\title{
An Optimal UAV Deployment Algorithm for Bridging Communication
}

\author{
Hanif Ullah*, Sally McClean*, Patrick Nixon ${ }^{\dagger}$, Gerard Parr ${ }^{\ddagger}$ and Chunbo Luo ${ }^{\S}$ \\ ${ }^{*}$ School of Computing and Information Engineering,Ulster University, Coleraine, UK \\ †Vice Chancellor's office, Ulster University, Coleraine, UK \\ ${ }^{\ddagger}$ School of Computing Sciences, University of East Anglia, Norwich Research Park, Norwich, UK \\ $\S$ Department of Computer Science, University of Exeter, Exeter, UK \\ Emails: ullah-h3@email.ulster.ac.uk,si.mcclean@ulster.ac.uk,paddy.nixon@ulster.ac.uk,g.parr@uea.ac.uk, c.luo@exeter.ac.uk
}

\begin{abstract}
In recent years, Unmanned Aerial Vehicles (UAVs) have attracted the attention of both the military and civilians because of their deployment in situations where part of the communication infrastructure is destroyed due to bomb blast, earthquake, flood, military operations or landslides. Also UAVs can be used in operations such as search and rescue, surveillance, forest fire monitoring, and border patrolling. Deployment of a UAV in a position where it can provide maximum coverage and high throughput is one of the vital problem that needs to be addressed. In this paper, we have proposed an optimal UAV deployment algorithm (OUDA) in order to bridge communication between two static nodes on the ground. In the proposed algorithm the UAV deploys to a position where it can provide the best communication facilities to both the nodes based on the received signal strength (RSS), and distance between nodes and UAV. Simulation results showed that the algorithm provides maximum throughput and low bit error rate (BER) once the UAV is fixed to an optimal position.
\end{abstract}

Keywords-Unmanned Aerial Vahicle (UAV), Bridging Communication, Optimal Deployment, Received Signal Strength (RSS), Bit Error Rate (BER), Disaster Management.

\section{INTRODUCTION}

Unmanned Aerial Vehicles (UAVs), also termed drones, are widely used for military applications suited for situations that are dull and dangerous. But in recent years UAVs have been used for many civil and commercial applications such as security and control, search and rescue operations, disaster management, crop management, communication, and land and border monitoring [1]. A UAV is basically an aircraft that can possibly operate without the intervention of any human being or crew, and because of this facility UAVs offers many advantages such as omitting the rescue parameters of the crew from the design not only saving space but also facilitating the UAV to be designed role and operation specific. Also, UAVs can be used in missions that are dangerous to the health of the crew. Examples of such missions are wind estimation [2], search and rescue operations [3], fire monitoring [4], [5], climate research [6], border patrolling and traffic monitoring [7], [8], and agricultural purposes [9]. In addition, UAVs can be used as communication relays in situations where part of the communication infrastructure is destroyed due to bomb-blast, earthquake or flood and there in no possibility to bridge communication between disjoint nodes on the ground
[10], [11]. Using UAVs as communication relays to bridge communication between nodes in any kind of disaster (bombblast, earthquake, flood etc.) is the main point of interest of this paper.

UAVs as communication relays have received much attention in recent years and a lot of work have been done in this area. Mozaffari, et al. analyzed the deployment of a UAV as a flying base station [12]. They used the idea to provide on-the-fly wireless communication facilities to a specific geographical area for a determined device-to-device communication network. The authors explained their problem considering two different scenarios with a static UAV and a mobile UAV. In scenario one, the authors derived the average coverage and sum-rate based on the UAV altitude and number of D2D users in the specified area, while in scenario two, the authors used the concept of a disk covering problem in order to calculate the minimum number of stop points to cover the entire area. The authors continue to say that the overall communication rate and coverage could be improved significantly if the UAV is precisely moved over the specified area [12].

Similarly, Jagun and Hailes [11] presented a dynamic positioning algorithm in order to bridge communication between participating nodes using the UAV as a relay. The authors claim that this approach could be used in real-life situations such as an earthquake and collecting data from distributed sensor nodes. The approach works in a way that a direct link can be established between nodes that are in the range of the UAV. A delay tolerant network (DTN) concept is used for nodes that are not in the communication range of the UAV for the sake of bridging communication. In the DTN approach the UAV flies over the participating nodes to collect, buffer and deliver data to and from the UAV. The authors also proposed a scheduling framework where they prioritised different nodes based on the frequency of visit and communication range in order to cover the entire set of distributed nodes [11].

Furthermore, in [13], the authors explored a communication system with some ground based terminals along with a network base station with a view to bridge communication between them using a UAV as a relay. The authors developed an algorithm for performance optimisation of the link between 
ground base terminals and relay. They also investigated the deployment of new UAV relays to the existing network in situation when the current UAV relay does not meet the minimum link requirements [13]. Moreover Morgenthaler, et al. [14] developed UAVNet, a flying wireless mesh network based on unmanned aerial vehicles (UAVs) connected through IEEE 802.11 s. The proposed system connects two end systems to communicate with each other through a single or multiple UAVs. The authors explained their work with different scenarios using two different positioning modes. Scenario one was carried out with a single UAV connecting to end systems located on the ground.

In this paper we investigate the problem of quickly deploying the UAV to a position where it can bridge communication between the participating nodes on the ground in such preceding application scenarios. The UAV will start flying towards the area hit by a disaster such as earthquake, flood, or bomb blast and will start transmitting hello/beacon messages at regular intervals. Once the nodes on the ground get the hello message, in response the nodes will send their ID and GPS position back to the UAV. The UAV will store this information in their connectivity metrics and start hovering in the immediate surrounding to find a position based on the received signal strength (RSS), and distance between the UAV and nodes in order to provide the optimal communication facilities to participating nodes on the ground.

The rest of the paper is structured as follows: Section II discusses the related work. Section III describes the system model. Section IV presents details concerning the proposed algorithm along with the experimental work. Section V discusses the results and discussion, while Section VI concludes the paper.

\section{RELATED WORK}

Many algorithms have been developed in order to optimally deploy a single UAV between two static nodes to facilitate communication between them. Jagun and Hailes [11], developed a greedy search algorithm and implemented it on a proportional-integral-derivative (PID) controller. The algorithm tries to achieve optimum communication between the participating nodes and instructs the UAV to move away or towards those participating nodes. The PID controller drives the UAV quickly in order to establish the communication link between nodes as soon as possible [11]. The algorithm optimally deploys the UAV based on balanced signal-to-noise (SNR) ratio, throughput and bit error rate (BER). To do so, the algorithm checks the SNR at different altitudes and once the SNR is balanced between the participating nodes, the UAV moves towards that particular position. The authors used SNR and throughput as input variables and the PID controller decides the next waypoint of the UAV based on these input variables. The algorithm instructs the UAV to move on ( $\mathrm{x}$, y) coordinates sending the echo messages at regular intervals and upon receiving any returning data from the participating nodes; the UAV tends to move towards that particular node. Similarly, the UAV calculates the maximum of throughput and SNR based on returning packets in order to find the most advantageous position where the UAV can serve all the participating nodes at the same time [11].

Furthermore, Han, et al. proposed an algorithm for optimizing the movement and location of UAVs in order to improve the connectivity of a wireless network [15]. The authors evaluated four different types of network connectivity cases, i.e. global message connectivity, worst-case connectivity, network bisection connectivity and k-connectivity. A twonode one-UAV scenario was addressed and different heuristic adaptive techniques were proposed for optimal UAV deployment [15]. In terms of global message, the two-node one-UAV problem is similar to the Steiner point problem from graph theory, where adding a node that can minimize the cost of a networks minimal spanning tree (MST) is termed a Steiner point. Similarly, for optimal UAV deployment, the authors consider the case of global messaging, worst-case connectivity and network bisection connectivity. A linear search algorithm was implemented in two dimensions in order to reduce the complexity of the gradient given in an equation is:

$$
g_{0}=\frac{d U\left(x_{0}, y_{0}\right)}{\left(d x_{0}\right)}+z \frac{d U\left(x_{0}, y_{0}\right)}{d y_{0}}
$$

The authors implemented their algorithm so that an initialization step is performed using either a heuristic or a random method in order to initialize the position of the UAV. In the iteration step, the authors calculate the gradient using equation (1) and then update the step index in consideration of optimizing the position of the UAV.

Additionally, Morgenthaler, et al. [14], addressed the same issue where they tried to bridge communications between two end systems using a single UAV. The authors developed a framework using the quadcopter based UAVs for the deployment of a flying wireless mesh network termed as UAVNet. The developed framework mainly controls the functionality of the UAV in terms of managing the deployed wireless mesh network [14]. The authors discussed different application scenarios where they are trying to establish communication between nodes (end systems) that are not connected directly or they are far from each other and have obstacles as well. A wireless mesh node is mounted on the UAV in order to send and receive traffic and to bridge communication. The UAV starts flying towards the first end system and sends ping messages at regular intervals. Once the first end system receives these ping messages, in response it sends its GPS position back to the UAV. Upon acknowledging the GPS position of the first end system, the UAV starts flying towards the second end system based on two searching modes i.e. manual and autonomous. In manual searching, the first end system will redirect the UAV toward the second end system, while in autonomous searching, the UAV calculates multiple waypoints on a spiral track and will start following a specific route in order to find the location of second end system. Once the UAV gets the GPS position of the second end system, it will deploy itself between the two end systems. The authors used a uav-controller that will calculate the mid-point of both 
end systems based on positioning modes i.e. location based positioning mode and signal strength positioning mode. In location based positioning mode, the uav-controller calculates the position of the UAV based on the submitted GPS positions of the two end systems, while in the signal strength positioning mode, the uav-controller also considers the received signal strength of both end systems in order to calculate a more precise position for the UAV [14].

Apart from algorithm-based techniques, some researchers have worked on simulation-based approaches where they considered aerial mast vs aerial bridge scenarios in the context of UAVs as a relay [16]. The authors evaluated two different strategies for bridging communication between two mobile ground nodes that are not in a line-of-sight with each other in an urban environment. In the aerial bridge scenario, a UAV is placed at an optimal position where it can receive the best signal strength from both the participating nodes on the ground. The optimal position could be the geometric centre of the nodes in a free space environment with line-of-sight, where the difference between the maximum received signal strength for both links will be less than a defined threshold. In the case of an aerial mast, the authors tried to maintain the position of the UAV above one of the ground nodes and then follow this node so as to maintain communication. Some of the problems with the navigation algorithm for the aerial bridge scenario were also addressed such as measuring the signal strength value because of the mobility of nodes and antenna radiation pattern is quite difficult. The simulation in both cases was carried out in Matlab using the Winner II propagation model with non-line-of-sight (NLoS) for air-to-ground channel. The performance of both scenarios was evaluated based on received signal power and distance between the UAV and the ground [16].

\section{SySTEM MODEL}

In this paper we proposed a model where a single UAV needs to be deployed between two static nodes on the ground as shown in Figure 1. Some of the key challenges associated with this case are deploying the UAV to an optimal position, resource monitoring for both UAV and nodes on the ground, and safe landing in the case of battery loss or mission termination. One of the important factors that should be considered in the case of wireless communication using a UAV as a relay is the path loss (PL) and propagation model. Path loss is a measure of the average radio frequencies (RF) attenuation at the receivers end suffered due to a transmitted signal [17] and can be denoted in decibels $(\mathrm{dB})$. Path loss can be expressed as in [18].

$$
P L(d B)=10 \log \frac{P_{t}}{P_{r}}
$$

where $P_{t}$ is the power of the transmitting antenna, while $P_{r}$ is the power of receiving antenna. In free space also termed as Line-of-Sight (LoS) communication, the electromagnetic wave propagates in a straight line and can be expressed by Friis free-

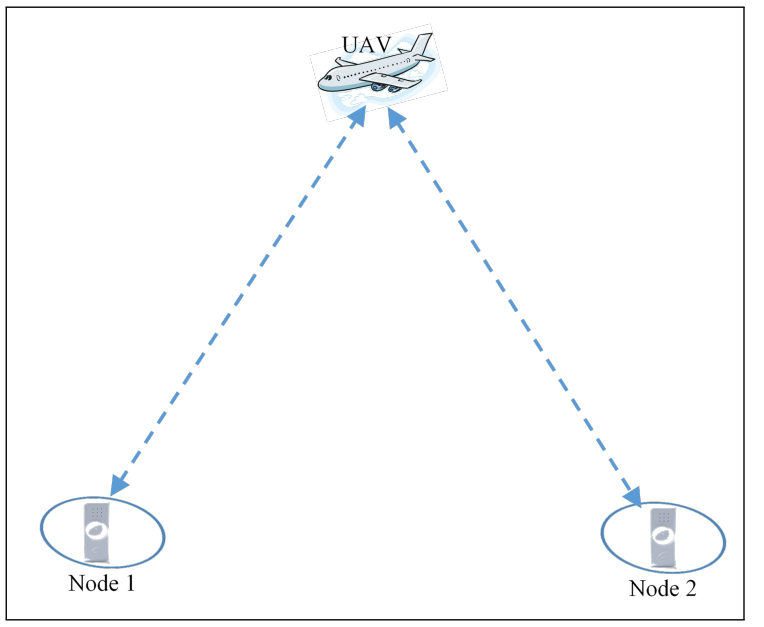

Fig. 1: Single UAV deployment between two static nodes

space equation [19]. A derivation of the following equation can be found in [20]

$$
P_{r}=\frac{P_{t} G_{t}\left(\theta_{t}, \phi_{t}\right) G_{r}\left(\theta_{r}, \phi_{r}\right) \lambda^{2}}{(4 \pi d)^{2}}
$$

where $P_{t}$ and $G_{t}$ are the power and gain of the transmitted antenna along with the elevation angle $\theta_{t}$ and azimuth angle $\phi_{t}$ respectively. The elevation angle normally ranges from $0^{\circ}$ to $180^{\circ}$, while the azimuth angle ranges from $0^{\circ}$ to $360^{\circ}$. $\lambda$ is the wavelength of the transmitted signal, while $d$ is the distance between the transmitting and receiving antenna. In wireless communication, the transmitted electrical signal in the transmitter is converted to electromagnetic waves by means of an antenna in order to propagate. The electromagnetic waves propagate at a speed of light expressed in meter/sec, with a frequency in $\mathrm{Hz}$, along with a wavelength, and can be written mathematically as follows:

$$
\lambda=\frac{c}{f}
$$

where $c$ is the speed of light and is equal to $3 * 10^{8} \mathrm{~m} / \mathrm{s}$, while $f$ is the frequency in Hz. substituting equation (3) in (4) yields:

$$
P_{r}=P_{t} G_{t}\left(\theta_{t}, \phi_{t}\right) G_{r}\left(\theta_{r}, \phi_{r}\right)\left(\frac{c}{4 \pi d f}\right)^{2}
$$

For digital signalling, we are normally dealing with energy per bit with respect to noise density at the receiver end. If we denote the data rate of the received signal by $R b_{r}$, and bandwidth of the receiver with $W$, then energy per bit with specified noise density can be written as

$$
\frac{E n_{b}}{N_{d}}=\left(P_{r}\right)\left(\frac{W}{R b_{r}}\right)
$$

Assuming that the channel is a one-bit channel i.e. $(W=1)$, then putting the value of $P_{r}$ from equation (5) we have:

$$
\frac{E n_{b}}{N_{d}}=\left(\frac{P_{t} G_{t}\left(\theta_{t}, \phi_{t}\right) G_{r}\left(\theta_{r}, \phi_{r}\right)}{R b_{r}}\right)\left(\frac{c}{4 \pi d f}\right)^{2}
$$




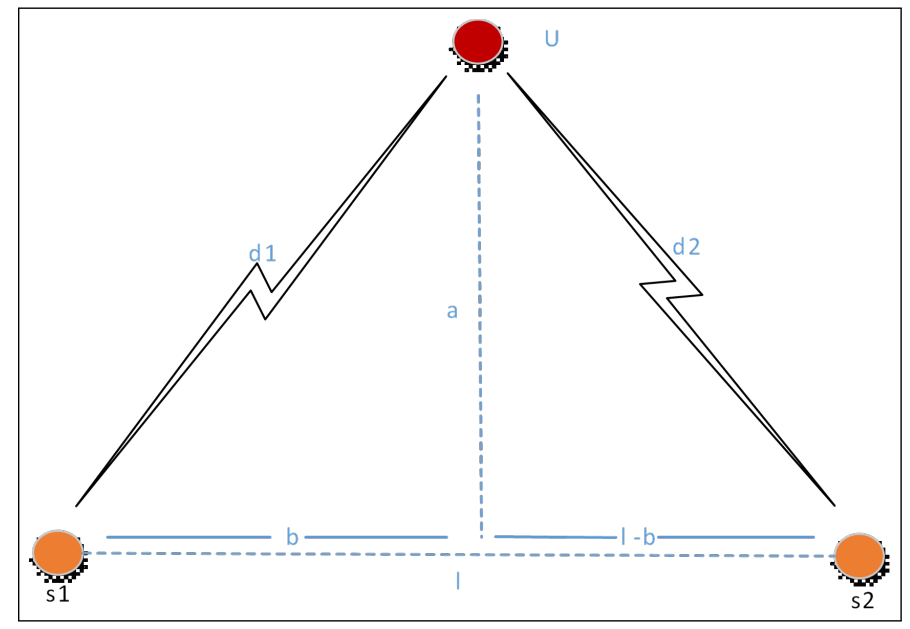

Fig. 2: A single UAV with two static nodes

Considering other factors of interference such as jitter, atmospheric loss, and delay, we can re-write the above equation as:

$$
\frac{E n_{b}}{N_{d}}=\left(\frac{P_{t} G_{t}\left(\theta_{t}, \phi_{t}\right) G_{r}\left(\theta_{r}, \phi_{r}\right)}{R b_{r}, I}\right)\left(\frac{c}{4 \pi d f}\right)^{2}
$$

Where $I$ is the interference because of the signal degradation and other loss factors. For successful communication between sender and receiver, the antenna mounted on node should have significant transmit power and gain in order to achieve the specified bit error rate (BER) at the receiving end. Similarly, by Figure 2 we can also derive $d_{1}$ (distance between node 1 (s1) and $\mathrm{UAV}(\mathrm{U})$ ) and $d_{2}$ (distance between node 2 (s2) and UAV (U)) given in equation (9) and (10) respectively.

$$
\begin{gathered}
d_{1}=\sqrt{b^{2}+a^{2}} \\
d_{2}=\sqrt{(l-b)^{2}+a^{2}}
\end{gathered}
$$

Where $l$ is the total distance between node 1 and 2, $a$ is the altitude of UAV, and $b$ is a fraction of the distance from node 1 to the centre of both nodes as shown in Figure 2 above. Now, we assume that a small scale fading between UAV and node will cause fluctuations in the signal power, so we will use the Rayleigh distribution method. In such a case we will suppose that equation (5) is equal to $\mathrm{P}$, where $\mathrm{P}$ is a deterministic factor as shown in the equation (11) below:

$$
P_{t} G_{t}\left(\theta_{t}, \phi_{t}\right) G_{r}\left(\theta_{r}, \phi_{r}\right)\left(\frac{c}{4 \pi d f}\right)^{2}=\frac{P}{d^{2}}
$$

Also, let $|h|$ denotes the fading (Rayleigh distribution) between UAV and node, then

$$
|h|^{2} \sim \frac{1}{\sigma_{\ell}^{2}} \exp \left(-\frac{|h|^{2}}{\sigma_{\ell}^{2}}\right)
$$

Since we know that $d_{1}=\sqrt{b^{2}+a^{2}}$ from equation (9), the signal power between node 1 and UAV, and node 2 and UAV can be calculated as follows:

$$
P_{1}=\frac{P\left|h_{1}\right|^{2}}{b^{2}+a^{2}}
$$

$$
P_{2}=\frac{P\left|h_{2}\right|^{2}}{(l-b)^{2}+a^{2}}
$$

In order to compute the full digital signal expression as shown in equation (15) below, we need to calculate the SNR between nodes and UAV as:

$$
y_{r}=\sqrt{P_{1}} \quad x+n
$$

Where $y_{r}$ is the received signal, $\sqrt{P_{1}}$ is the channel coefficient and power, $x$ denotes the signal transmitted (unit power), and $n$ shows the noise. To calculate the SNR between nodes and UAV, we will derive the following equations:

$$
S N R_{1}=\frac{\left|\sqrt{P_{1}}\right|^{2}\left|x_{1}\right|^{2}}{|n|^{2}} \text { where } n \sim N\left(0, \sigma_{n}^{2}\right)
$$

By putting the value of $n$ will yield the following equations:

$$
\begin{aligned}
& S N R_{1}=\frac{\left|\sqrt{P_{1}}\right|^{2}}{\sigma_{n}^{2}}=\frac{P_{1}}{\sigma_{n}^{2}} \\
& S N R_{2}=\frac{\left|\sqrt{P_{2}}\right|^{2}}{\sigma_{n}^{2}}=\frac{P_{2}}{\sigma_{n}^{2}}
\end{aligned}
$$

In some cases, we can also write the transmitted SNR in the form of $\frac{P_{t}}{\sigma_{n}^{2}}$. Also in bandwidth (represented by W) limited channel, we will have the capacity as $W \log \left(1+\frac{P_{t}}{W \sigma_{n}^{2}}\right)$, and for simplicity we will assume that $W=1$ (a one-bit channel). The instant capacity for a fading channel can then be calculated as:

$$
C_{1}=\log \left(1+S N R_{1}\right)=\log \left(1+\frac{P_{1}\left|h_{1}\right|^{2}}{\left(x^{2}+a^{2}\right) \sigma_{n}^{2}}\right)
$$

Since the channel is fading (fluctuating), we are interested in seeing the average capacity that can be calculated using the following equation:

$$
\overline{C_{1}}=\int_{0}^{\infty} C_{1} p_{1}\left(\left|h_{1}\right|^{2}\right) d\left|h_{1}\right|^{2}
$$

where $p\left(\left|h_{1}\right|^{2}\right.$ ), is the probability density function (PDF) which is used in the case of a fading channel. By substituting the value of $C_{1}$ in equation (19) will result the equation below.

$$
\begin{array}{r}
\overline{C_{1}}=\int_{0}^{\infty} \log \left(1+\frac{P_{1}\left|h_{1}\right|^{2}}{\left(x^{2}+a^{2}\right) \sigma_{n}^{2}}\right) \\
\frac{1}{\sigma_{\ell}^{2}} \exp \left(-\frac{\left|h_{1}\right|^{2}}{\sigma_{\ell}^{2}}\right) d\left|h_{1}\right|^{2}
\end{array}
$$

In equation (21) above, only $\left|h_{1}\right|^{2}$ is the variable factor, while the rest are treated as deterministic factors during the integration. Equation (21) can be integrated by simplifying some of the notations such as,

$$
y=\frac{P_{1}}{\left(x^{2}+a^{2}\right) \sigma_{n}^{2}}, z=\left|h_{1}\right|^{2}
$$

By substituting the values of $\mathrm{y}$ and $\mathrm{z}$ in equation (21) will yield:

$$
\overline{C_{1}}=\int_{0}^{\infty} \log (1+y z) \frac{1}{\sigma_{\ell}^{2}} \exp \left(-\frac{z}{\sigma_{\ell}^{2}}\right) d z
$$


Equation (23) can further be integrated by using the integration by parts method as follows:

$$
\begin{array}{r}
u=\log (1+y z), d v=\frac{1}{\sigma_{\ell}^{2}} \exp \left(-\frac{z}{\sigma_{\ell}^{2}}\right) d z \\
d u=\frac{y}{1+y z}, v=-\exp \left(-\frac{z}{\sigma_{\ell}^{2}}\right) \\
=\left(-\log (1+y z) \exp \left(-\frac{z}{\sigma_{\ell}^{2}}\right)\right) \\
\left.\right|_{z=0} ^{z=\infty}+\int_{0}^{\infty} \frac{y}{1+y z} \exp \left(-\frac{z}{\sigma_{\ell}^{2}}\right) d z
\end{array}
$$

The first part of the equation (26) becomes zero as we know that the exponent at infinity is equal to zero. Also we know that $\log 1=0$. Equation (26) can therefore be re-written as,

$$
=\int_{0}^{\infty} \frac{y}{1+y z} \exp \left(-\frac{z}{\sigma_{\ell}^{2}}\right) d z
$$
(27):

Further simplifying and assigning new variables to equation

$$
r=1+y z, \text { so } z=\frac{r-1}{y}, d z=\frac{d r}{y}
$$

By substituting the value of $r$ in equation (27) will yield equation (29) as follows.

$$
\begin{aligned}
& =\int_{1}^{\infty} \frac{1}{r} \exp \left(-\frac{r-1}{y \sigma_{\ell}^{2}}\right) d r \\
= & e^{\frac{1}{y \sigma_{\ell}^{2}}} \int_{1}^{\infty} \frac{1}{r} \exp \left(-\frac{r}{y \sigma_{\ell}^{2}}\right) d r
\end{aligned}
$$

Further assigning a variable to a part of the equation (29) for the sake of simplicity,

$$
\frac{r}{y \sigma_{\ell}^{2}}=t
$$

By putting the value of equation (30) in (29) will produce the new equation as follows.

$$
=e^{\frac{1}{y \sigma_{\ell}^{2}}} \int_{\frac{1}{y \sigma_{\ell}^{2}}}^{\infty} \frac{e^{-t}}{t} d t
$$

The exponential integral function is a function that can be defined as $\operatorname{Ei}(k)=-\int_{-k}^{\infty} \frac{e^{-t}}{t} d t$ from [21]. Using the concept of [21] will yield equation (32) as follows.

$$
=-e^{\frac{1}{y \sigma_{l}^{2}}} \operatorname{Ei}\left(-\frac{1}{y \sigma_{l}^{2}}\right)
$$

By putting the value of y from equation (22) will yield equation (33) as follows.

$$
\overline{C_{1}}=\operatorname{Ei}\left(-\frac{\left(x^{2}+a^{2}\right) \sigma_{n}^{2}}{P_{1} \sigma_{\ell}^{2}}\right) \exp \left(\frac{\left(x^{2}+a^{2}\right) \sigma_{n}^{2}}{P_{1} \sigma_{\ell}^{2}}\right)
$$

From the equation above, the UAV position can be controlled and calculated by using the variable $x$ and $a$, where $x$ is the transmitted signal and $a$ is the altitude/distance between UAV and nodes.

\section{THE PROPOSED ALGORITHM}

In this section we describe the algorithm that we have developed for the optimal deployment of a UAV in order to bridge communication between two static nodes on the ground. The algorithm is termed Optimal UAV Deployment Algorithm (OUDA). The algorithm works by the UAV first flying towards the affected area in order to search for targets. Originally, we have considered the targets as sensor nodes but they may be human beings with some smart devices or may be some other objects. Once the UAV reaches the area, it will start searching for nodes. Two different searching procedures are used in our algorithm i.e. Spiral search and Ladder search. The detail about each of these searching procedures is given below.

\section{A. Spiral Search}

In this type of searching the UAV flies to the corner/centre of the target area and starts searching for the nodes following a spiral route as shown in Figure 3 below. The UAV moves towards the corner of the targeted area and then flies to the centre in order to starts the searching process. The searching time can be increased or decreased by increasing or decreasing the number of laps. The algorithm calculates the searching time, number of nodes found, and the time the UAV takes to reach the optimal position.

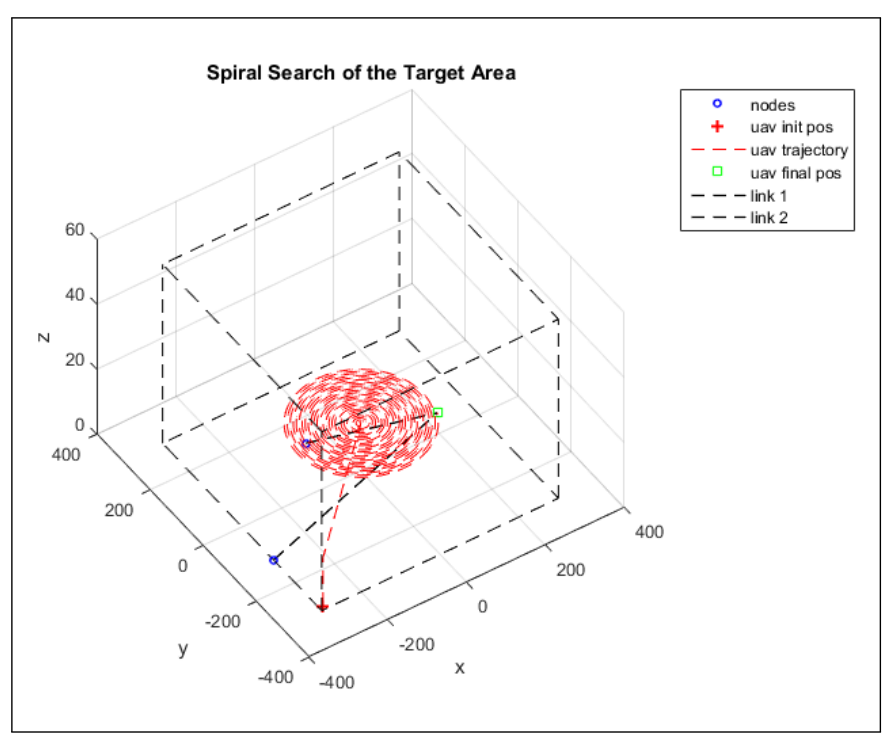

Fig. 3: Spiral search with UAV trajectory

\section{B. Ladder Search}

As its name implies, ladder search is a kind of search where the UAV searches the entire area just like the ladder steps. In this case the UAV moves towards the corner of the target area and starts the searching process as shown in Figure 4. Just like the spiral search, the number of steps/phases can be increased or decreased in order to increase or decrease the searching time. The algorithm calculates the same parameters as it calculates in the case of spiral search. 


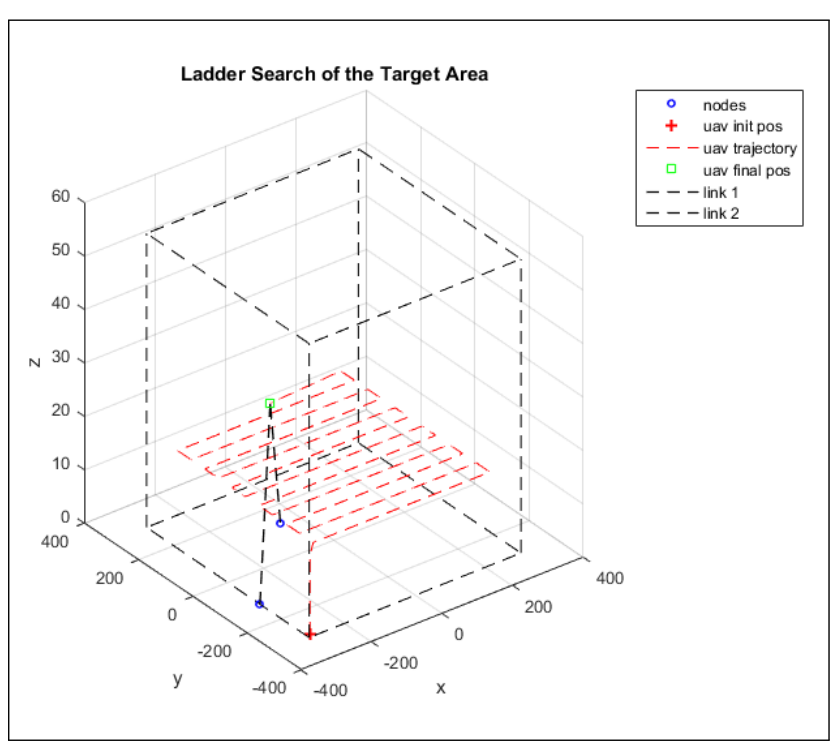

Fig. 4: Ladder search with UAV trajectory

In both of the above searching modes, spiral search provide good results in terms of finding the nodes and time to search the entire area. Once the UAV finishes the searching process, it moves towards the optimal position. Optimal position of the UAV is calculated based on the geographical existence of nodes (distance of nodes from the UAV d) and received signal strength/transmitted signal power $(\mathrm{x})$ from the nodes. Once the UAV reaches the optimal/best position, it stays there for the entire simulation time in order to provide communication facilities to nodes on the ground. During the search phase the algorithm also calculates the bit error rate (BER) for both links between the nodes and UAV as shown in Figure 5. The BER is erratic when the UAV is searching for nodes because of the fluctuation in signal, but once the UAV moves to the optimal position the BER becomes consistent.

\section{RESUlts AND Discussion}

In this section, some of the simulation results in terms of graphs will be explained in order to verify the effectiveness of OUDA. The algorithm is developed and simulated in Matlab with the simulation parameters tabulated in table 1 . The entire simulation is carried out with two nodes which are distributed randomly and one UAV, where the minimum distance between the nodes is one meter.

The path loss exponent is 2 because the propagation is in free space and there is a direct line of sight (LOS) communication between the nodes and UAV. During the search phase, the altitude of the UAV is kept to 15 meters with a maximum speed of $15 \mathrm{~m} / \mathrm{s}$ (meter per second), but once the search phase is finished, the UAV moves to an optimal position with either a high altitude or low altitude depending on the signal transmit power from the nodes. Initially we have transmitted 100 packets with 128 bits/packet in order to calculate the BER between nodes and UAV.

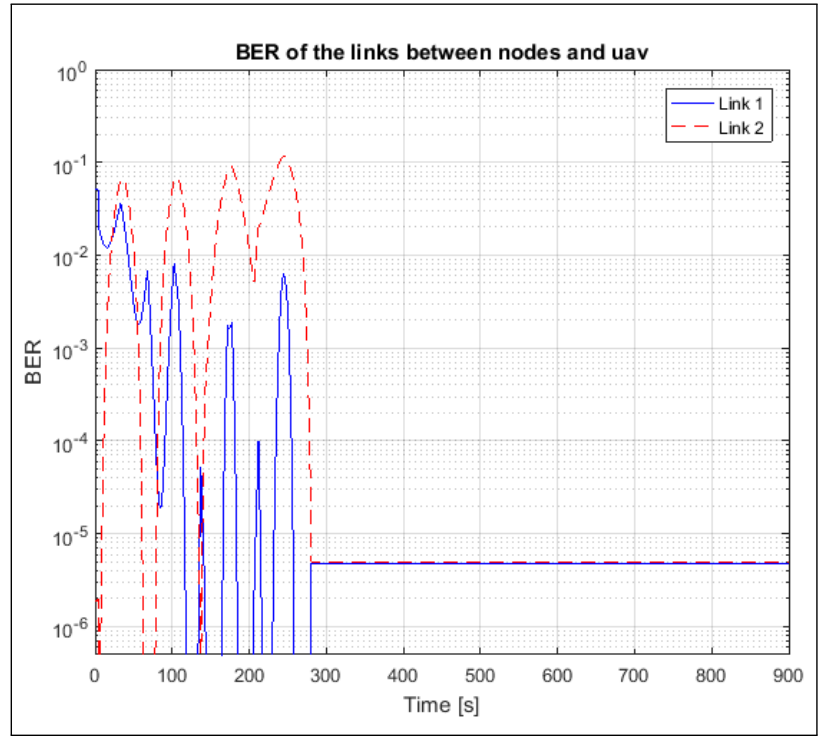

Fig. 5: BER of the Links between nodes and UAV

TABLE I:

\section{SIMULATION PARAMETERS FOR OUDA}

\begin{tabular}{|l|l|}
\hline Simulation Parameters & Value \\
\hline No. of Nodes & 2 \\
\hline No. of UAVs & 1 \\
\hline Node transmit power & $-10 \mathrm{db}$ \\
\hline Minimum Distance & 1 meter \\
\hline Pathloss exponent & 2 \\
\hline Shaddowing & $0 \mathrm{db}$ \\
\hline UAV maximum speed & $15 \mathrm{~m} / \mathrm{s}$ \\
\hline Search altitude & $15 \mathrm{~meters}$ \\
\hline Packet size & $256 \mathrm{Kbps}$ \\
\hline
\end{tabular}

Based on the simulation parameters results are obtained in terms of graphs for RSSI, SNR, and BER that are explained below. Due to space limitations, we have only included the graphs for RSSI and BER.

\section{A. Received Signal Strength Indicator (RSSI)}

RSSI is a measurement of how well a UAV can hear a signal from the ground nodes. RSSI is normally measured in decibels with a negative value. The graph in Figure 6 shows the value of RSS for both node 1 and 2. During the search phase the RSSI between UAV and nodes is inconsistent as the UAV is moving around to find the nodes on ground by either using a spiral search or ladder search method. Once the searching phase is over and the UAV moves to the optimal position, the RSSI becomes consistent as shown in Figure 6 below.

\section{B. Bit Error Rate (BER)}

Bit error rate or BER is basically the rate at which the error occurs in a transmission system during the communication. The graph in Figure 7 shows the bit error rate between UAV and nodes. BER is basically the rate at which the error occurs in a transmission system between transmitter and receiver. In the case of a good signal to noise ratio, the BER will be very 


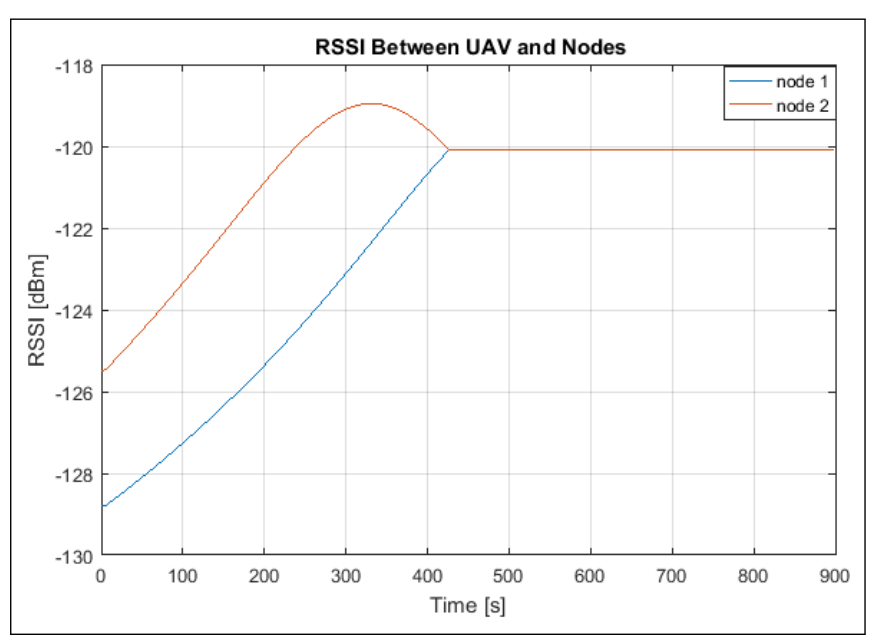

Fig. 6: RSSI for node 1 and 2

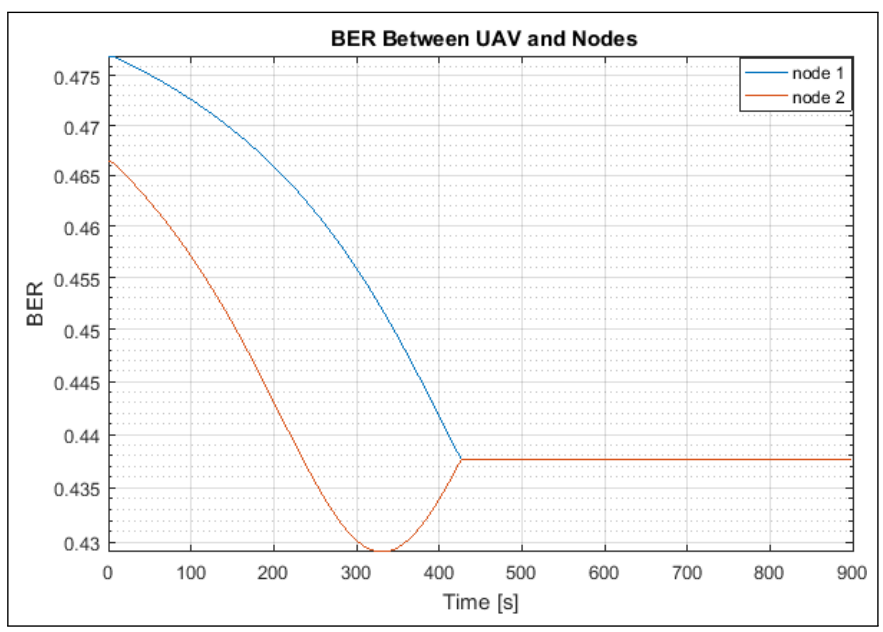

Fig. 7: Bit error rate with respect to time

small and sometimes negligible. During the search phase, the BER is varying because of the fluctuation in the signal, but once the UAV finds the optimal position, the BER becomes stable as shown in the graph in Figure 7.

\section{CONCLUSION}

In this paper we have proposed and investigated the performance of an optimal UAV deployment algorithm (OUDA) in order to deploy a single UAV between two static nodes on the ground for developing communication infrastructure. The UAV is deployed to an optimal position based on RSSI, and distance between the participating nodes and UAV. The algorithm performs well in terms of low BER, which makes the algorithm suitable for real time applications in disaster management.

In the future, we are planning to extend our algorithm to a group of static or moving nodes employing clustering algorithms. The use of multiple UAVs to provide communication facilities to a larger area is also planned. Moreover, comparing our algorithm with other algorithms and producing more experimental results from real and simulated data is also part of our future work.

\section{REFERENCES}

[1] P. B. Charlesworth, "A game theoretic approach to coordinating unmanned aerial vehicles with communications payloads," Ph.D. dissertation, School of Computer Science \& Informatics, Cardiff University, UK. Available at: http://orca.cf.ac.uk/75627/1/2015charlesworthpbPhD.PDF, 2015.

[2] A. Cho, J. Kim, S. Lee, and C. Kee, "Wind estimation and airspeed calibration using a uav with a single-antenna gps receiver and pitot tube," IEEE Transactions on Aerospace and Electronic Systems, vol. 47, no. 1, pp. 109-117, January 2011.

[3] J. George, P. B. Sujit, and J. B. Sousa, "Search strategies for multiple uav search and destroy missions," Journal of Intelligent \& Robotic Systems, vol. 61, pp. 355-367, Jan. 2011.

[4] V. Ambrosia, S. Wegener, J. Brass, and S. Schoenung, "The uav western states fire mission: Concepts, plans and developmental advancements," Proceedings of the AIAA 3rd Unmanned Unlimited Conference, 2004.

[5] C. Barrado, R. Messeguer, J. Lopez, E. Pastor, E. Santamaria, and P. Royo, "Wildfire monitoring using a mixed air-ground mobile network," Pervasive Computing, IEEE, vol. 9, pp. 24-32, 2010.

[6] W. Bolton, "Operational experience with uav payloads for climate research applications," 2nd AIAA Unmanned Unlimited Systems, Technologies, and Operations, 2003.

[7] K. Ro, J.-S. Oh, and L. Dong, "Lessons learned: Application of small uav for urban highway traffic monitoring," 45th AIAA Aerospace Sciences Meeting and Exhibit, pp. 2007-596, 2007.

[8] Z. Sun, P. Wang, M. C. Vuran, M. A. Al-Rodhaan, A. M. Al-Dhelaan, and I. F. Akyildiz, "Bordersense: Border patrol through advanced wireless sensor networks," Ad Hoc Networks, vol. 9, pp. 468-477, 2011.

[9] H. Xiang and L. Tian, "Development of a low-cost agricultural remote sensing system based on an autonomous unmanned aerial vehicle (uav)," Biosystems Engineering, vol. 108, pp. 174-190, 2011.

[10] 1. Bekmezci, O. K. Sahingoz, and a. Temel, "Flying ad-hoc networks (fanets): A survey," Ad Hoc Networks, vol. 11, pp. 1254-1270, 2013.

[11] K. Jagun and S. Hailes, "Scheduling uavs to bridge communications in delay-tolerant networks using real-time scheduling analysis techniques," System Integration (SII), 2014 IEEE/SICE International Symposium on, pp. 363-369, 2014.

[12] M. Mozaffari, W. Saad, M. Bennis, and M. Debbah, "Unmanned aerial vehicle with underlaid device-to-device communications: Performance and tradeoffs," IEEE Transactions on Wireless Communications, vol. 15, no. 6, pp. 3949-3963, June 2016.

[13] X. Yang, L. M. Alvarez, and T. Bruggemann, "A 3d collision avoidance strategy for uavs in a non-cooperative environment," Journal of Intelligent \& Robotic Systems, vol. 70, pp. 315-327, 2013.

[14] S. Morgenthaler, T. Braun, Z. Zhongliang, T. Staub, and M. Anwander, "Uavnet: A mobile wireless mesh network using unmanned aerial vehicles," Globecom Workshops (GC Wkshps), 2012 IEEE, pp. 1603$1608,2012$.

[15] Z. Han, A. L. Swindlehurst, and K. J. R. Liu, "Optimization of manet connectivity via smart deployment/movement of unmanned air vehicles,' IEEE Transactions on Vehicular Technology, vol. 58, pp. 3533-3546, 2009.

[16] C. B. Moussa, F. Gagnon, O. Akhrif, and S. Gagne, "Aerial mast vs aerial bridge autonomous uav relay: A simulation-based comparison," 2014 6th International Conference on New Technologies, Mobility and Security (NTMS), pp. 1-5, 2014.

[17] T. K. Sarkar, Z. Ji, K. Kim, A. Medouri, and M. Salazar-Palma, "A survey of various propagation models for mobile communication," Antennas and Propagation Magazine, IEEE, vol. 45, pp. 51-82, 2003.

[18] H. L. Bertoni, Radio Propagation for Modern Wireless Systems. Prentice Hall Professional Technical Reference, 1999.

[19] A. K. Mylin, "A communication link reliability study for small unmanned aerial vehicles," University of Kentucky Master Theses, 2007.

[20] C. A. Balanis, Antenna theory, analysis and design. John Wiley and Sons, 2016.

[21] A. Jeffrey and D. Zwillinger, Table of Integrals, Series, and Products (Seventh Edition). Boston: Academic Press, 2007. [Online]. Available: http://www.sciencedirect.com/science/article/pii/B9780080471112500030 